To appear in The Astrophysical Journal, February 1, 1997

\title{
MULTIFREQUENCY RADIO OBSERVATIONS OF THE GRAVITATIONAL LENS SYSTEM MG 0414+0534
}

\author{
C. A. Katz, C. B. Moore円, \& J. N. Hewitt \\ Department of Physics and Research Laboratory of Electronics \\ Room 26-331, Massachusetts Institute of Technology \\ Cambridge, Massachusetts, 02139 \\ Electronic mail: ckatz@maggie.mit.edu,cmoore@maggie.mit.edu, jhewitt@maggie.mit.edu
}

\begin{abstract}
The four-image gravitational lens system MG 0414+0534 was observed with the VLA at 1.4, 5, 8, 15, and $22 \mathrm{GHz}$. The 15 and $22 \mathrm{GHz}$ images reveal structure in the components which is consistent with that seen at other wavelengths. There was no detection of other extended emission, nor were there detections of the lensing galaxy or the "component $x$ " seen in optical images. Detections of polarized emission show that the fractional polarized intensity is $\lesssim 1 \%$. The observed properties of MG0414 suggest that it is a GHz-peaked spectrum source.
\end{abstract}

Subject headings: gravitational lensing — radio continuum: general — quasars: individual (MG 0414+0534)

\section{Introduction}

Since the discovery of the "double quasar" $0957+561$ by Walsh et al. (1979), work on gravitational lensing has grown to address many topics in astrophysics. From observations of multi-component systems and Einstein rings to theoretical studies of the implications of lensing statistics on cosmology, the study of gravitational lenses provides us with a unique probe of many phenomena. Lenses may function as "cosmic telescopes" for studying background sources; modeling lens gravitational potentials allows us to explore the mass distribution in galaxies and clusters; measuring time delays between the light curves for different images of the same source allows us to estimate the values of cosmological parameters. A review of some applications of gravitational lenses is given by Blandford \& Narayan (1992). To engage profitably in such pursuits requires gravitational lens systems with well-measured characteristics. MG 0414+0534 (also

\footnotetext{
${ }^{1}$ Present address: Kapteyn Astronomical Institute, Postbus 800, 9700 AV Groningen, The Netherlands
} 
4C05.19 and PKS0411+05; hereafter MG0414) is a gravitational lens system that is very bright at radio wavelengths and has optical and infrared counterparts.

MG0414 was discovered in the MIT-Green Bank survey (Bennett et al. 1986). It was selected from the survey in a gravitational lens search by Hewitt (1986) and Hewitt et al. (1989). It was later studied in more detail by Hewitt et al. (1992) and Katz \& Hewitt (1993). The later observations showed that MG0414 displays many of the characteristics expected in a gravitational lens system: morphology typical of lensing by an elliptical potential, similar spectral indices of the components, and frequency-independent flux density ratios of the components. While the optical flux ratios reported by Schechter \& Moore (1993), Falco (1993), and Angonin-Willaime et al. (1994) differ significantly from those in the radio, Witt, Mao, \& Schechter (1995) conclude from simulations of microlensing in the MG0414 system that the differences between the radio and optical flux ratios are consistent with microlensing models with plausible physical parameters. In addition, the observation of significant reddening of the quasar images has fueled speculation that the flux ratio differences are due at least in part to obscuration by dust (Lawrence et al. 1995; Vanderriest, Angonin-Willaime, \& Rigaut 1996).

Other evidence in favor of gravitational lensing includes the discovery of the lensing galaxy by Schechter \& Moore (1993), the measurement of similar optical spectra for the A and B components (Angonin-Willaime et al. 1994), and the measurement of the source redshift of 2.639 (Lawrence et al. 1995).

Herein we describe VLAf observations which were planned with several objectives in mind: high angular resolution, to reveal structure in the components which would be useful for constraining lens models; sensitivity to extended emission, to map any extended structure, also useful in modeling the lens and in examining the nature of the background source; measurement of polarization structure, to provide information about the background source and to measure Faraday rotation; multifrequency observations, to measure the spectral indices of the components; and sensitivity to faint objects, to search for a fifth image and the lensing galaxy itself.

\section{Observations and Data Analysis}

\subsection{Observations}

MG0414 was observed with the A configuration of the VLA at $15 \mathrm{GHz}$ on 1992 November 18 and 1992 December 15, at 1.4, 5, 8, and $15 \mathrm{GHz}$ on 1993 January 15, at 8 and $22 \mathrm{GHz}$ on 1994 April 1, and with the B configuration at $15 \mathrm{GHz}$ on 1993 March 9. A summary of the observations is given in Table 1. The 15 GHz runs in 1992 and on 1993 March 9 were part

\footnotetext{
${ }^{2}$ The VLA is part of the National Radio Astronomy Observatory, which is operated by Associated Universities, Inc., under cooperative agreement with the National Science Foundation
} 
of a longer term monitoring program, and were selected because they were the longest of the monitoring runs that took place when the sky was clear. During the 1993 January 15 observing, thick cloud cover and a snowstorm complicated the calibration, especially at $15 \mathrm{GHz}$. At that frequency, 200 minutes were spent on MG0414, but about one-third of the data were discarded because of very poor phase stability. At the other frequencies, phase stability was satisfactory. Weather conditions during the 1994 observations were ideal; the VLA's reference pointing mode (Perley 1995) was used at $22 \mathrm{GHz}$. In all but the monitoring observations the delay center was placed two arc seconds east of the center of the system, rather than directly at the center, to avoid introducing any biases that might affect our search for a fifth image or radio emission from the lensing galaxy.

Table 1. Summary of Observations of MG $0414+0534$

\begin{tabular}{|c|c|c|c|c|}
\hline $\begin{array}{l}\text { Date } \\
\text { (UT) }\end{array}$ & $\begin{array}{l}\text { Central } \\
\text { Frequency }^{\mathrm{a}} \\
\quad(\mathrm{GHz})\end{array}$ & $\begin{array}{c}\text { Integration } \\
\text { Time }\end{array}$ & Resolution $^{\mathrm{b}}$ & $\begin{array}{l}\text { Map RMS } \\
(\mu \mathrm{Jy} / \text { beam })\end{array}$ \\
\hline $92 \operatorname{Nov} 18^{\mathrm{c}}$ & 14.940 & $10^{\mathrm{m}}$ & $0.14^{\prime \prime} \times 0.13^{\prime \prime},-27^{\circ}$ & 400 \\
\hline 92 Dec $15^{c}$ & 14.940 & $22^{\mathrm{m}}$ & $0.13^{\prime \prime} \times 0.12^{\prime \prime},+32^{\circ}$ & 280 \\
\hline \multirow[t]{4}{*}{$93 \mathrm{Jan} 15^{\mathrm{c}}$} & 1.415 & $25^{\mathrm{m}}$ & $1.23^{\prime \prime} \times 1.07^{\prime \prime},-12^{\circ}$ & 610 \\
\hline & 4.860 & $40^{\mathrm{m}}$ & $0.33^{\prime \prime} \times 0.31^{\prime \prime},-6^{\circ}$ & 200 \\
\hline & 8.440 & $40^{\mathrm{m}}$ & $0.24^{\prime \prime} \times 0.19^{\prime \prime},+6^{\circ}$ & 120 \\
\hline & 14.940 & $70^{\mathrm{m}}$ & $0.11^{\prime \prime} \times 0.10^{\prime \prime},-23^{\circ}$ & 250 \\
\hline 93 Mar $9^{\mathrm{d}}$ & 14.940 & $20^{\mathrm{m}}$ & $0.69^{\prime \prime} \times 0.37^{\prime \prime},-46^{\circ}$ & 340 \\
\hline \multirow[t]{2}{*}{94 Apr $1^{\mathrm{c}}$} & 8.440 & $5^{\mathrm{m}}$ & $0.24^{\prime \prime} \times 0.22^{\prime \prime},-2^{\circ}$ & 130 \\
\hline & 22.460 & $95^{\mathrm{m}}$ & $0.08^{\prime \prime} \times 0.08^{\prime \prime}$ & 330 \\
\hline
\end{tabular}

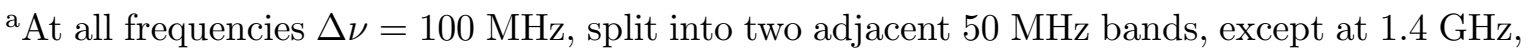
where the central frequencies were 1365 and $1465 \mathrm{MHz}$.

${ }^{\mathrm{b}} \mathrm{FWHM}$ of beam major and minor axes and position angle of major axis measured north through east

${ }^{\mathrm{c}}$ VLA A-configuration

${ }^{\mathrm{d}}$ VLA B-configuration 


\subsection{Calibration and Mapping}

In all data sets but one the flux density scales were set by reference to $3 \mathrm{C} 48$. In the $15 \mathrm{GHz}$ data of 1993 January 15, the flux density scale was set by reference to 3C286 because 3C48 was resolved on even the shortest interferometric baselines. Because 3C48 is resolved on all baselines at $22 \mathrm{GHz}$, observations of it at that frequency were bracketed by short scans on the phase calibrator $0133+476$, allowing the creation of a model of $3 \mathrm{C} 48$ which was used to set the flux density scale.

The VLA antenna gains at all frequencies were determined from short observations of the calibrator source 0420-014. This calibrator was also used to determine the feed polarizations of the antennas. In the monitoring runs, 3C147 was used to fix the linear polarization position angle. All calibration and mapping was done with NRAO's Astronomical Image Processing System (AIPS). The maps shown here were produced after several iterations of phase self-calibration, followed in some cases by one iteration of amplitude self-calibration. The self-calibration significantly improved the dynamic range of the images. Deconvolution was performed with the AIPS task MX, restricting clean components to small regions at the positions of the four objects.

Figures 1 through 5 show, respectively, the $1.4 \mathrm{GHz}$ through $22 \mathrm{GHz}$ maps produced from the 1993 January 15 observations. The dynamic ranges in the best maps are limited to about 2500:1 (peak intensity:RMS noise level), which is much smaller than the dynamic range of 10,000:1 expected if radiometer noise is the limiting factor. Since well-known methods for improving the dynamic ranges of VLA maps proved unhelpful (see for example Perley 1989), we suspect that the deconvolution procedure itself limited the dynamic range (see Briggs \& Cornwell 1994, Briggs 1995). The combination of the VLA dirty beam and the particular morphology of MG0414 appears to exacerbate this effect; tests performed with noiseless models of MG0414 confirm that this is indeed the case. Further work in investigating deconvolution algorithms is needed to improve the dynamic ranges of VLA maps of MG0414.

The most notable feature in the high-resolution maps is the extended structure: component B is extended to the southeast, and components A1 and A2 are extended toward each other, with a faint connecting "bridge" of flux density approximately $2 \mathrm{mJy}$ visible at $15 \mathrm{GHz}$. The extensions are consistent with the structure seen in a 5 GHz MERLIN image (Garrett et al. 1992), and with that seen in the Hubble Space Telescope (HST) image of Falco, Lehár, \& Shapiro (1996). Our data provide upper limits on the surface brightness of a fifth image of the background source and

on any emission associated with the lensing galaxy. If we take these limits to be 10 times the RMS surface brightness in each map, we find they are 6.1, 2.0, 1.2, 2.5, and 3.3 mJy at 1.4, 5, 8, 15 and $22 \mathrm{GHz}$, respectively. Expressed as a fraction of the flux density of component B the limits are $0.017,0.013,0.013,0.038$, and 0.072 . We do not detect "component $x$," the sixth object west of component B detected at optical wavelengths by Schechter \& Moore (1993), and confirmed by Angonin-Willaime et al. (1994) and Falco et al. (1996).

The 1.4 GHz observation was intended to reveal any low surface brightness extended emission which is likely to have a steep radio spectrum and to give a $10 \sigma$ detection of $70 \mathrm{mJy}$ distributed 
uniformly over a circle of radius $10^{\prime \prime}$. An area the size of the primary beam (about $31^{\prime}$ at $1.4 \mathrm{GHz}$ ) was mapped, but no extended emission smaller than $38^{\prime \prime}$ (the maximum angular scale to which this configuration of the VLA is sensitive) was found, even in maps in which the short baselines were given large weights.

\subsection{Radiometry and Astrometry}

Absolute and relative radiometry of MG0414 (referred to component B) are presented in Tables 2 \& 3, respectively. Except for the monitoring observations and the $8 \mathrm{GHz}$ observation on 1994 April 1, the flux density uncertainties reported in Table 2 were computed by comparing three effects: 1) the scatter in the amplitudes on unresolved baselines of the calibrated uv-data for the primary flux calibrator, 2) the uncertainty incurred in the transfer of the antenna gain amplitudes from the primary flux calibrator to the phase calibrator (0420-014), and 3) the scatter in the ensemble of flux density measurements made by splitting the dataset into many short time segments and mapping each separately. In all cases, 1) was negligible with respect to 2). Since 2) and 3) are independent, the flux density uncertainties were found by adding them in quadrature. For the $15 \mathrm{GHz}$ observation on 1993 January 15 which used 3C286 to set the flux scale, an additional $2 \%$ uncertainty was added in quadrature reflecting the unknown systematic difference in the flux density scale between 3C48 and 3C286. The difference in elevation between the flux calibration source and the phase calibrator was less than $6^{\circ}$ at 15 and $22 \mathrm{GHz}$, so elevation-dependent opacity differences should not make a significant contribution to the error. For the monitoring observations, we estimate the error from the scatter in flux estimates over the whole monitoring dataset $(3.5 \%)$. The uncertainties in the relative flux densities reported in Table 3 were computed from scatter of the $\mathrm{A} 1 / \mathrm{B}, \mathrm{A} 2 / \mathrm{B}$, and $\mathrm{C} / \mathrm{B}$ ratios in the segmented data described above. The $\mathrm{C} / \mathrm{B}$ ratio at $8 \mathrm{GHz}$ is significantly different from that reported in Katz \& Hewitt (1993). However, since the data used in Katz \& Hewitt (1993) had a number of problems, and the ratios in Table 3 are consistent with those measured from other radio observations (e.g. Hewitt et al. 1992), we conclude that the difference in ratios cannot be regarded as reliable evidence for variability.

Astrometry of MG0414 is presented in Table 4 . Since the measured positions were consistent at all five frequencies, we show only the positions determined from the $22 \mathrm{GHz}$ observations, which had the highest resolution. The position of component B was found by fitting a two-dimensional gaussian. The uncertainties in the absolute position of B were taken to be $0.1^{\prime \prime}$, the highest accuracy expected under normal observing conditions (Perley 1995). The uncertainties in the positions of components $\mathrm{A} 1, \mathrm{~A} 2$, and $\mathrm{C}$ relative to $\mathrm{B}$ were calculated from the scatter in the segmented data (described above). The positions reported in Table 4 are consistent with those reported by Falco et al. (1996) from HST observations.

\footnotetext{
${ }^{3}$ Note that variability in the source would make this an over-estimate of the error.
} 
Table 2. Absolute radiometry of MG 0414+0534.

\begin{tabular}{|c|c|c|c|c|c|c|}
\hline Frequency & $\begin{array}{c}\text { Date of } \\
\text { Observation }\end{array}$ & A1 & $\mathrm{A} 2$ & B & $\mathrm{C}$ & Total \\
\hline $1.4 \mathrm{GHz}$ & 93 Jan 15 & \multicolumn{2}{|c|}{$1644 \pm 8^{\mathrm{a}}$} & $349 \pm 6$ & $125 \pm 6$ & $2118 \pm 11$ \\
\hline $5 \mathrm{GHz}$ & 93 Jan 15 & $401 \pm 8$ & $362 \pm 8$ & $156 \pm 4$ & $58 \pm 2$ & $976 \pm 15$ \\
\hline \multirow[t]{2}{*}{$8 \mathrm{GHz}$} & 93 Jan 15 & $245 \pm 2$ & $220 \pm 2$ & $95 \pm 1$ & $36 \pm 1$ & $596 \pm 4$ \\
\hline & 94 Apr 1 & $242 \pm 1$ & $217 \pm 1$ & $93 \pm 1$ & $37 \pm 1$ & $598 \pm 13$ \\
\hline \multirow[t]{4}{*}{$15 \mathrm{GHz}$} & 92 Nov 18 & $162 \pm 6$ & $142 \pm 6$ & $64 \pm 3$ & $23 \pm 1$ & $391 \pm 11$ \\
\hline & 92 Dec 15 & $151 \pm 6$ & $134 \pm 5$ & $59 \pm 2$ & $23 \pm 1$ & $367 \pm 11$ \\
\hline & 93 Jan 15 & $170 \pm 4$ & $150 \pm 4$ & $66 \pm 2$ & $24 \pm 2$ & $411 \pm 10$ \\
\hline & 93 Mar 9 & $145 \pm 6$ & $129 \pm 5$ & $56 \pm 2$ & $21 \pm 1$ & $351 \pm 10$ \\
\hline $22 \mathrm{GHz}$ & 94 Apr 1 & $117 \pm 6$ & $103 \pm 5$ & $46 \pm 3$ & $17 \pm 2$ & $282 \pm 11$ \\
\hline
\end{tabular}

Flux densities are in mJy referenced to $3 \mathrm{C} 48$.

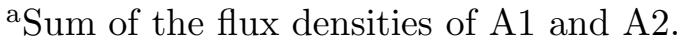

Table 3. Relative radiometry of MG $0414+0534$.

\begin{tabular}{rccccc}
\hline \hline & $\begin{array}{c}\text { Date of } \\
\text { Observation }\end{array}$ & A1 & A2 & B & C \\
& & & & & \\
\hline $1.4 \mathrm{GHz}$ & 93 Jan 15 & \multicolumn{2}{c}{$4.786 \pm 0.046^{\mathrm{a}}$} & 1.000 & $0.344 \pm 0.020$ \\
$5 \mathrm{GHz}$ & 93 Jan 15 & $2.589 \pm 0.021$ & $2.325 \pm 0.016$ & 1.000 & $0.372 \pm 0.003$ \\
$8 \mathrm{GHz}$ & 93 Jan 15 & $2.573 \pm 0.003$ & $2.309 \pm 0.003$ & 1.000 & $0.384 \pm 0.001$ \\
$15 \mathrm{GHz}$ & 93 Jan 15 & $2.567 \pm 0.010$ & $2.286 \pm 0.009$ & 1.000 & $0.389 \pm 0.003$ \\
$22 \mathrm{GHz}$ & 94 Apr 1 & $2.522 \pm 0.026$ & $2.221 \pm 0.022$ & 1.000 & $0.396 \pm 0.006$ \\
\hline
\end{tabular}

$\mathrm{a}(\mathrm{A} 1+\mathrm{A} 2) / \mathrm{B}$ 


\subsection{Polarimetry}

All data, except those taken at $15 \mathrm{GHz}$ on 1993 January 15, were analyzed for the polarization properties of the source. Maps of the Stokes Q, U, and V parameters were computed. In the $1.4 \mathrm{GHz}$ and $5 \mathrm{GHz}$ data of 1993 January 15, there are nominal detections of polarized emission, giving a polarization fraction of approximately 0.003 for A1 and A2. However, the positions of the polarized emission peaks are significantly different $\left(\sim 0.15^{\prime \prime}\right)$ from the positions of the total intensity peaks, raising the question of the accuracy of the polarization measurements. The position offset we measure implies an uncertainty in the fractional polarization of 0.006 (Roberts, Wardle, \& Brown 1994); therefore, we conclude that the 1993 January 15 detections are spurious. Since the observing conditions on 1993 January 15 were less than optimal, and the absence of data from an unpolarized source on that date precludes correcting for the time-variable polarization response of the VLA, we derive information on the polarization from the other datasets only. Polarized emission was detected from $\mathrm{A} 1$ and $\mathrm{A} 2$ at $15 \mathrm{GHz}$ in the monitoring runs; for A1, the measured polarization fraction is $0.0126 \pm 0.0011$ with position angle $-46^{\circ} \pm 2^{\circ}$ (measured north through east). For A2 the polarization fraction is $0.0123 \pm 0.0009$ with position angle $-52^{\circ} \pm 6^{\circ}$. The errors are based on the scatter in the data. In the $8 \mathrm{GHz}$ data of 1994 April 1 there were marginal detections of polarized emission from A1 and A2 with polarization fractions less than 0.01, but the signal-to-noise ratios in the maps are so low that we are unable to reliably estimate the polarization fractions or the position angles.

Table 4. $22 \mathrm{GHz}$ astrometry of MG 0414+0534.

\begin{tabular}{ccc}
\hline \hline Component & \multicolumn{1}{c}{$\Delta \alpha\left(^{\prime \prime}\right)$} & \multicolumn{1}{c}{$\Delta \delta\left(^{\prime \prime}\right)$} \\
& & \\
\hline $\mathrm{A} 1$ & $+0.5876^{\prime \prime} \pm 0.0003^{\prime \prime}$ & $-1.9341^{\prime \prime} \pm 0.0003^{\prime \prime}$ \\
$\mathrm{A} 2$ & $+0.7208^{\prime \prime} \pm 0.0003^{\prime \prime}$ & $-1.5298^{\prime \prime} \pm 0.0003^{\prime \prime}$ \\
$\mathrm{B}$ & 0. & 0. \\
$\mathrm{C}$ & $-1.3608^{\prime \prime} \pm 0.0007^{\prime \prime}$ & $-1.6348^{\prime \prime} \pm 0.0008^{\prime \prime}$ \\
\hline
\end{tabular}

Positions are relative to component $\mathrm{B}$, which is located at RA $04^{\mathrm{h}} 14^{\mathrm{m}} 37.7275^{\mathrm{s}} \pm 0.1^{\prime \prime}$, Dec $+05^{\circ} 34^{\prime} 44.276^{\prime \prime} \pm 0.1^{\prime \prime}(\mathrm{J} 2000)$ 


\subsection{Spectral Indices}

For each component, a spectral model of the form $S=S_{o} \nu^{-\alpha}$ was fit to the $5,8,15$, and $22 \mathrm{GHz}$ flux density measurements. The 5,8 , and $15 \mathrm{GHz}$ flux densities were taken from the simultaneous measurements of 1993 January 15. The results are shown in Figure 6. Including the 1.4 GHz points in the fits made them unacceptable; we take this as an indication that the spectral index $\alpha$ changes below $5 \mathrm{GHz}$. The spectral indices for the four components are consistent; averaging them yields an estimate for the source spectral index of $\alpha=0.80 \pm 0.02$.

\section{Discussion}

Quadruple-image lens systems are much better suited for gravitational potential modeling than double-image lens systems because the additional images provide more constraints. However, even quadruple-image systems do not provide unique lens models when only the position and flux density of each component are measured (Gorenstein, Shapiro, \& Falco 1988; Kochanek 1991; Wambsganss \& Paczyński 1994). Resolving structure in the images is required to constrain lens models. The maps of MG0414 at 15 and $22 \mathrm{GHz}$ show structure on a scale comparable to the sizes of the synthesized beams, making possible an improvement over the modeling effort of Kochanek (1991) and Hewitt et al. (1992). Modeling work is underway using both the VLA data reported here and VLBI data (Ellithorpe, Hewitt, \& Kochanek 1996). Our failure to detect "component $x$ " is not surprising in light of the fact that it is resolved in the HST image, and thus appears to be a galaxy (Falco et al. 1996).

If the source is an isotropic radiator, the radio spectral luminosity density implied by the observed flux density of component $\mathrm{B}$ is $\sim 10^{29} h^{-2} \mathrm{erg} \mathrm{s}^{-1} \mathrm{~Hz}^{-1}$ in the rest frame of the source (assuming $H_{0}=100 \mathrm{~h} \mathrm{~km} \mathrm{sec}{ }^{-1} \mathrm{Mpc}^{-1}, q_{0}=0.5$, and $\Lambda=0$ ). The magnification of the B image is not well constrained by existing lens models (Hewitt et al. 1992; Kochanek 1991; Witt et al. 1995). Values as large as 30 have been predicted, which would reduce the inferred intrinsic luminosity by the same factor. Even after correcting for the magnification, however, MG0414 has an intrinsic luminosity comparable to those of powerful radio galaxies and quasars. Only a very small fraction of the radio emission detected with the VLA is resolved, indicating a source size smaller than $1 h^{-1} \mathrm{kpc}$ (assuming $H_{0}=100 h \mathrm{~km} \mathrm{sec}^{-1} \mathrm{Mpc}^{-1}, q_{0}=0.5, \Lambda=0$, and unit magnification).

At the low resolution of the single-dish survey of Condon, Broderick, \& Seielstad (1991) the object appears as a double source; the brighter of the two components is at the position of the MG0414 quasar images. The fainter of the two is not seen in the $1.4 \mathrm{GHz}$ data in this work, implying a source size greater than $38^{\prime \prime}$. If the two radio components were physically associated, their angular separation of $12^{\prime}$ would correspond to a linear distance of $2.7 h^{-1} \mathrm{Mpc}$ at the source, at the high end of the distribution of radio source sizes (Fanaroff \& Riley 1974). However, there is no evidence in the NRAO VLA Sky Survey (Condon et al. 1993) for radio emission between the two sources, and it is unlikely that they are physically associated. 
Since the initial studies of MG0414 by Hewitt et al. (1992), the nature of the background source has been an issue because of its unusually red optical spectrum. Observations in several wavebands reported by Lawrence et al. (1995) have led them to conclude that the source is a quasar made to look unusual by the passage of the light rays through several magnitudes of extinction due to dust in the lensing galaxy. However, in the HST image of Falco et al. (1996) there is an arc of emission connecting component B to the A1-A2 component pair. The arc is significantly less red than the image cores. Thus they argue that if the arc and core sources have the same intrinsic color, and the arc-core separation is small, then the reddening is intrinsic to the core source rather than due to the lensing galaxy.

In Figure 0 we plot MG0414 flux density measurements from this work and from the literature. The radio spectrum turns over at low frequencies, characteristic of many high redshift compact objects. The small linear size and the peak of the radio spectrum near $1 \mathrm{GHz}$ suggest that MG0414 is an example of a GHz-peaked spectrum (GPS) radio source. The GPS sources form a class closely related to, and perhaps a subclass of, the compact steep spectrum (CSS) sources. The GPS sources have sub-kiloparsec sizes and spectral turnovers near $1 \mathrm{GHz}$, while the CSS sources have sizes of 1-10 kpc and spectral turnovers at one to several hundred $\mathrm{MHz}$ (Fanti et al. 1990, Kapahi 1981, Peacock \& Wall 1982, Heckman et al. 1994). The GPS sources are strongly correlated with high redshifts and large radio luminosities (Gopal-Krishna, Patnaik, \& Steppe 1983); MG0414 has both properties. The spectral index of MG0414 below the turnover frequency is $\sim 0.5$, intermediate to the ranges seen for GPS and CSS sources. Also, the low polarization fraction of MG0414 is consistent with the properties of these classes. Interestingly, another gravitational lens, 0218+357, has also been identified as a GPS source (O'Dea, Baum, \& Stanghellini 1991).

One possible explanation for the low polarization fraction is beam depolarization, which occurs when the linear polarization angle varies over a scale smaller than that of the synthesized beam. Assuming $H_{0}=100 h \mathrm{~km} \mathrm{~s}^{-1} \mathrm{Mpc}^{-1}, q_{0}=0.5, \Lambda=0$, unit magnification, and a likely lensing galaxy redshift of 0.5 (Kochanek 1992), the $5 \mathrm{GHz}$ beamwidth of $0.3^{\prime \prime}$ corresponds to a linear distance of $1.05 h^{-1} \mathrm{kpc}$; at the source redshift of $2.639,0.3^{\prime \prime}$ corresponds to a linear distance of $1.13 h^{-1} \mathrm{kpc}$. Therefore, in order to reduce the polarization fraction by $50 \%$, the rotation measure must change by about $200 \mathrm{rad} \mathrm{m}^{-2}$ over a distance of approximately $1.1 \mathrm{kpc}$. This arises naturally in models of GPS sources, but requires a lensing galaxy with unusual properties in order to produce the required depolarization as well as the observed optical reddening (Schechter 1995).

\section{Conclusions}

We have resolved some structure in components $\mathrm{A} 1, \mathrm{~A} 2$, and $\mathrm{B}$, at 15 and $22 \mathrm{GHz}$ which is consistent with the arc seen in the HST image of Falco et al. (1996). We have not detected emission from any other extended sources, from the lensing galaxy, or from "component $x$." It is unlikely that the observed structure will suffice to impose strong constraints on models of the 
radial mass distribution of the lens. Further work should address the deconvolution-imposed dynamic range limit in the maps, to reach the theoretical sensitivity limit and possibly resolve more low surface-brightness structure.

The spectral indices of the components at frequencies above $1.4 \mathrm{GHz}$ are consistent with each other. Averaging them yields a best estimate for the source spectral index of $\alpha=0.80 \pm 0.02$.

The fractional polarized flux density from MG0414 is very low, and is detected at the threshold where our polarization calibration becomes uncertain. To exploit the polarization of the source for time delay measurements or other studies, a program designed for more careful calibration of the polarization properties of the VLA will be required.

MG $0414+0534$ is a powerful high redshift quasar in which the effects of obscuration in the source (or less likely, in the lens) are extremely important. The source spectral peak at approximately $1 \mathrm{GHz}$, the compact structure, the high redshift, the large radio luminosity, and the low polarization fraction are all consistent with the GHz-peaked spectrum (GPS) sources. GPS sources are thought to be AGN which are confined to a very small space by an exceptionally dense circumnuclear gas. If the background source in MG0414 is indeed a GPS source, then the gravitational lens may provide an unusual opportunity to study a member of the GPS class through a "cosmic telescope." We are presently processing VLBA data which will address this possibility.

We wish to thank Rick Perley for his advice on VLA observing techniques, and Pauline McMahon for her help with the actual observations. Thanks are also due Jim Condon for providing us with an early map from the NRAO VLA Sky Survey. We are grateful to Emilio Falco for providing HST maps of MG0414, and for his useful suggestions. Finally, we thank the anonymous referee for helpful comments. This work was supported by a David and Lucile Packard Fellowship in Science and Engineering, a National Science Foundation Presidential Young Investigator Award, and the M.I.T. Class of 1948.

\section{REFERENCES}

Angonin-Willaime, M., Vanderriest, C., Hammer, F., \& Magain, P. 1994, A\&A, 281, 388

Bennett, C.L., Lawrence, C.R., Burke, B.F., Hewitt, J.N., \& Mahoney, J.H. 1986, ApJS, 61, 1

Blandford, R.D., \& Narayan, R. 1992, in ARA\&A, eds. G. Burbidge et al. (Palo Alto, CA: Annual Reviews Inc.), 315

Bolton, J.G., Savage, A., \& Wright, A.E. 1979, Australian J. Phys. Suppl., 46, 1

Briggs, D.S., \& Cornwell, T.J. 1994, in IAU Symposium \#158, Very High Angular Resolution Imaging, eds. J.G. Robertson, W.J. Tango (Dordrecht: Kluwer Academic Publishers)

Briggs, D.S. 1995, New Mexico Institute of Technology Ph.D. Thesis 
Condon, J.J., Broderick, J.J., \& Seielstad, G.A. 1991, AJ, 102, 2041

Condon, J.J., Cotton, W.D., Greisen, E.W., Perley, R.A., Yin, Q.F., Broderick, J.J. 1993, BAAS, $183,64.02$

Ellithorpe, J.D., Hewitt, J.N., \& Kochanek, C.S. 1996, in preparation

Falco, E.E. 1993, in Gravitational Lenses in the Universe, eds. J. Surdej et al. (Liège, Belgium: Université de Liège, Institut d'Astrophysique), 127

Falco, E.E., Lehár, J., \& Shapiro, I.I. 1996, ApJ, submitted

Fanaroff, B.L., \& Riley, J.M. 1974, MNRAS, 167, 31

Fanti, R., Fanti, C., Schilizzi, R.T., Spencer, R.E., Rendong, N. 1990, A\&A, 231, 333

Garrett, M.A., Patnaik, A.R., Muxlow, T.W.B., Wilkinson, P.N., \& Walsh, D. 1992, in Sub-arcsecond Radio Astronomy, eds. R.J. Davis \& R.S. Booth (Cambridge: Cambridge University Press), 146

Gopal-Krishna, MR., Patnaik, A.R., \& Steppe, H. 1983, A\&A, 123, 107

Gorenstein, M.V., Shapiro, I.I., \& Falco, E.E. 1988, ApJ, 327, 693

Gower, J.F.R., Scott, P.F., \& Wills, D. 1967, MmRAS, 71, 49

Gregory, P.C., \& Condon, J.J. 1991, ApJS, 75, 1011

Griffith, M.R., Wright, A.E., Burke, B.F., Ekers, R.D. 1995, ApJS, 97, 347

Heckman, T.M., O’Dea, C.P., Baum, S.A., \& Laurikainen, E. 1994, ApJ, 428, 65

Hewitt, J.N. 1986, M.I.T. Ph.D. Thesis

Hewitt, J.N., Burke, B.F., Turner, E.L., Schneider, D.P., Lawrence, C.R., Langston, G.I., \& Brody, J.P. 1989, in Lecture Notes in Physics, Vol. 330, Gravitational Lenses, eds. J.M. Moran, J.N. Hewitt, \& K.Y. Lo (Berlin: Springer-Verlag), 147

Hewitt, J.N., Turner, E.L., Lawrence, C.R., Schneider, D.P., \& Brody, J.P. 1992, AJ, 104, 968

Kapahi, V.K. 1981, A\&AS, 43, 381

Katz, C.A., \& Hewitt, J.N. 1993, ApJ, 409, L9

Kochanek, C.S. 1991, ApJ, 373, 354

Kochanek, C.S. 1992, ApJ, 384, 1

Large, M.I., Mills, B.Y., Little, A.G., Crawford, D.F., \& Sutton, J.M. 1981, MNRAS, 194, 693

Lawrence, C.R., Elston, R., Januzzi, B.T., \& Turner, E.L. 1995, AJ, 110, 2570

O’Dea, C.P., Baum, S.A., \& Stanghellini, C. 1991, ApJ, 380, 66

Peacock, J.A., \& Wall, J.V. 1982, MNRAS, 198, 843

Perley, R.A. 1989, in Synthesis Imaging in Radio Astronomy, eds. Perley, R.A., Schwab, F.R., \& Bridle, A.H., (San Francisco: Astronomical Society of the Pacific), 287 
Perley, R.A. 1995, VLA Observational Status Summary

Pilkington, J.D.H., \& Scott, P.F. 1965, MmRAS, 69, 183

Roberts, D.H., Wardle, J.F.C., \& Brown, L.F. 1994, ApJ, 427, 71

Schechter, Paul L. 1995, private communication

Schechter, Paul L. \& Moore, Christopher B. 1993, AJ, 105, 1

Vanderriest, C., Angonin-Willaime, M.-C., \& Rigaut, F. 1996, in IAU Symposium \#173, Astrophysical Applications of Gravitational Lensing, eds. C.S. Kochanek \& J.N. Hewitt (Dordrecht: Kluwer Academic Publishers), 353

Walsh, D., Carswell, R.F., \& Weymann, R.J. 1979, Nature, 279, 381

Wambsganss, J., \& Paczyński, B. 1994, AJ, 108, 1156

White, R.L., \& Becker, R.H. 1992, ApJS, 79, 331

Witt, Hans J., Mao, S., \& Schechter, Paul L. 1995, ApJ, 443, 18 


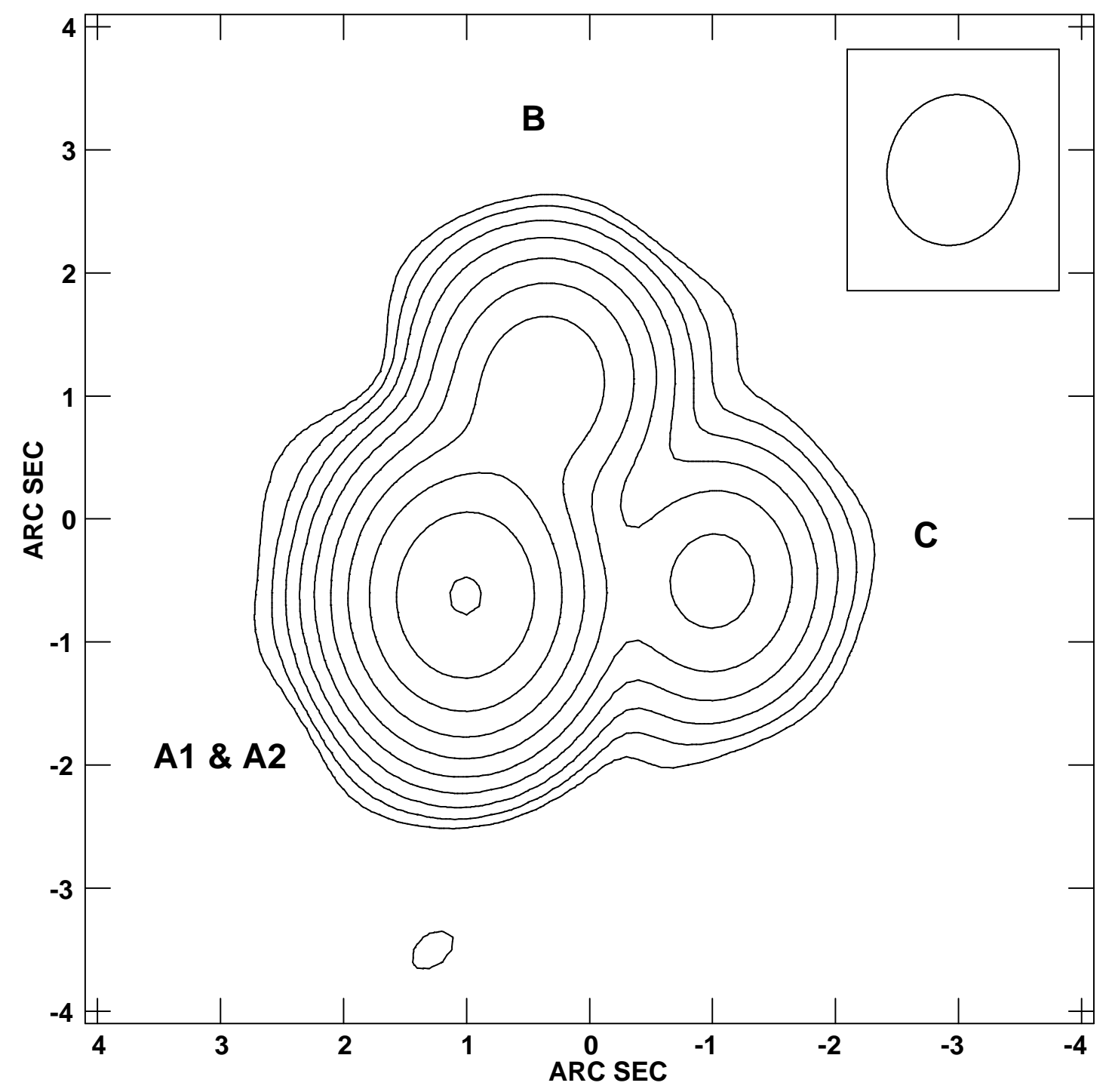

Fig. 1.- Contour plot of $1.4 \mathrm{GHz}$ map of MG $0414+0534$ on 93 Jan 15. Contour levels are $-0.1875 \%, 0.1875 \%, 0.375 \%, 0.75 \%, 1.5 \%, 3 \%, 6 \%, 12 \%, 24 \%, 48 \%$, and $96 \%$ of the peak intensity of $1.524 \mathrm{Jy} /$ beam. The RMS noise level is $610 \mu \mathrm{Jy} /$ beam. The box in the upper right corner shows the beam FWHM ellipse. The map is centered at RA $04^{h} 14^{m} 37.708^{s}$, Dec $+05^{\circ} 34^{\prime} 43.10^{\prime \prime}$ (J2000). 


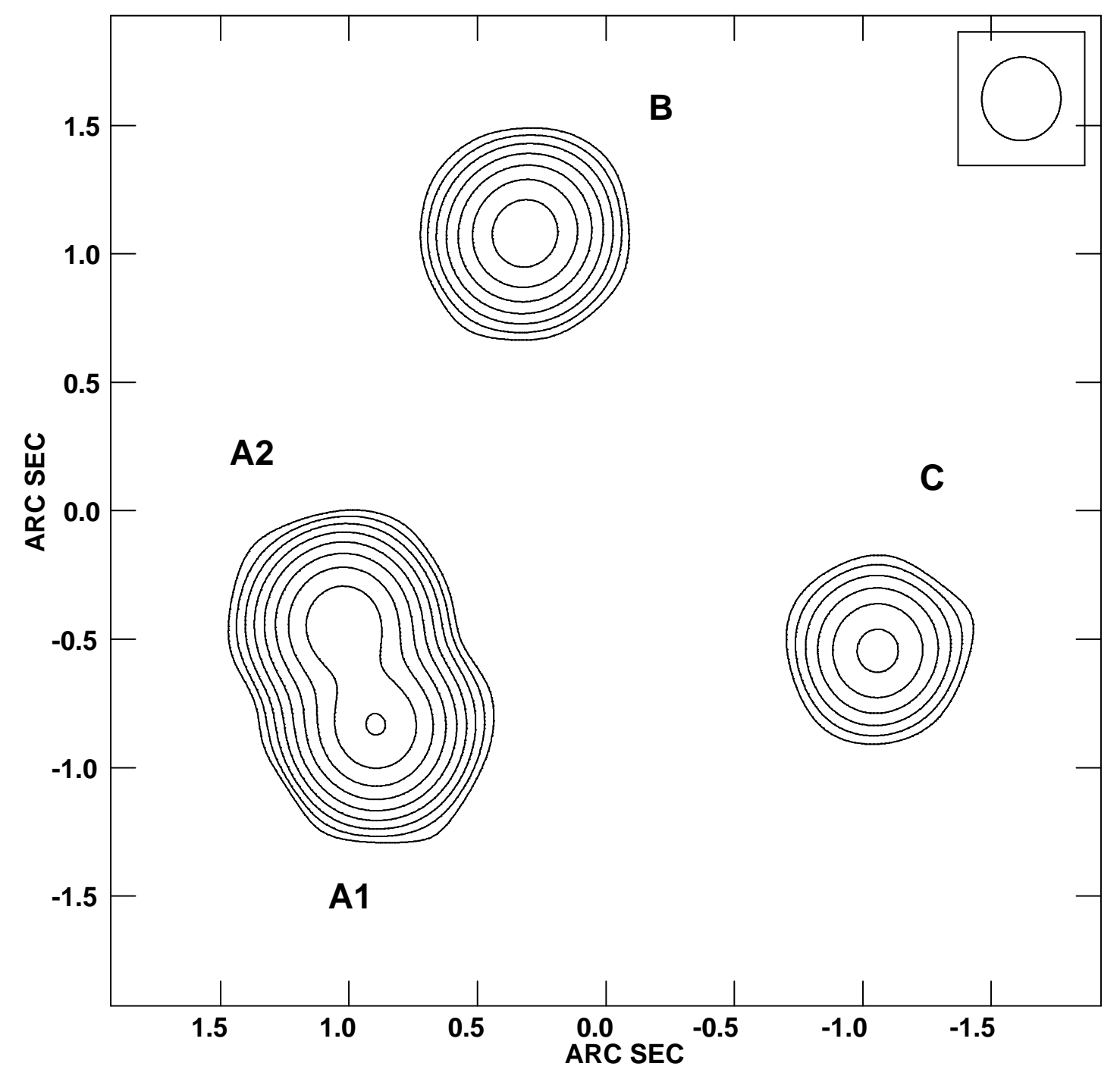

Fig. 2.- Contour plot of $5 \mathrm{GHz}$ map of MG $0414+0534$ on 93 Jan 15. Contour levels are $-0.375 \%$, $0.375 \%, 0.75 \%, 1.5 \%, 3 \%, 6 \%, 12 \%, 24 \%, 48 \%$, and $96 \%$ of the peak intensity of $398 \mathrm{mJy} / \mathrm{beam}$. The RMS noise level is $200 \mu \mathrm{Jy} /$ beam. The box in the upper right corner shows the beam FWHM ellipse. The map is centered at RA $04^{h} 14^{m} 37.707^{s}$, Dec $+05^{\circ} 34^{\prime} 43.19^{\prime \prime}$ (J2000). 


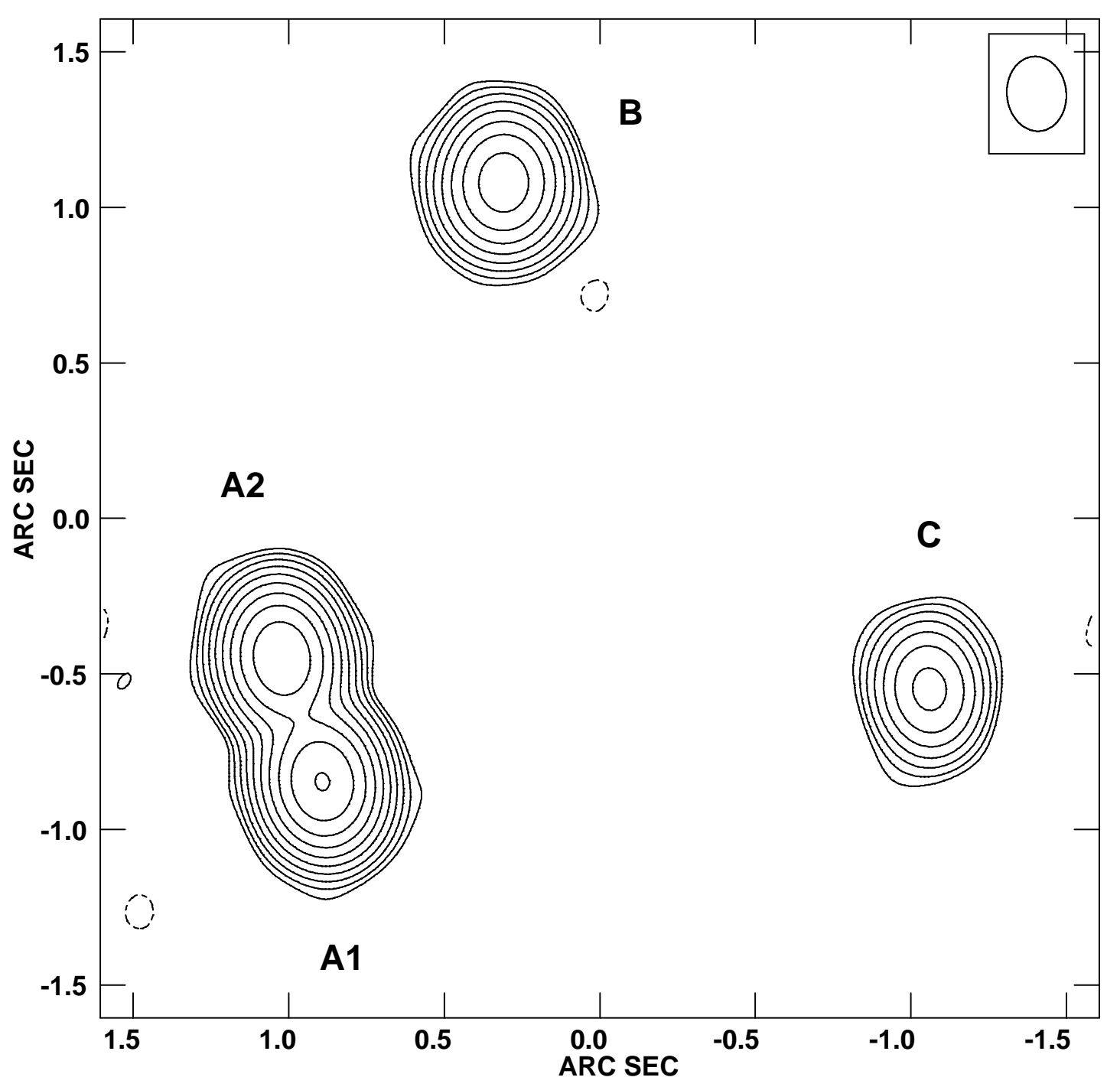

Fig. 3.- Contour plot of $8 \mathrm{GHz}$ map of $\mathrm{MG} 0414+0534$ on 93 Jan 15. Contour levels are $-0.1875 \%, 0.1875 \%, 0.375 \%, 0.75 \%, 1.5 \%, 3 \%, 6 \%, 12 \%, 24 \%, 48 \%$, and $96 \%$ of the peak intensity of $238 \mathrm{mJy} /$ beam. The RMS noise level is $120 \mu \mathrm{Jy} / \mathrm{beam}$. The box in the upper right corner shows the beam FWHM ellipse. The map is centered at RA $04^{h} 14^{m} 37.708^{s}$, Dec $+05^{\circ} 34^{\prime} 43.19^{\prime \prime}$ (J2000). 


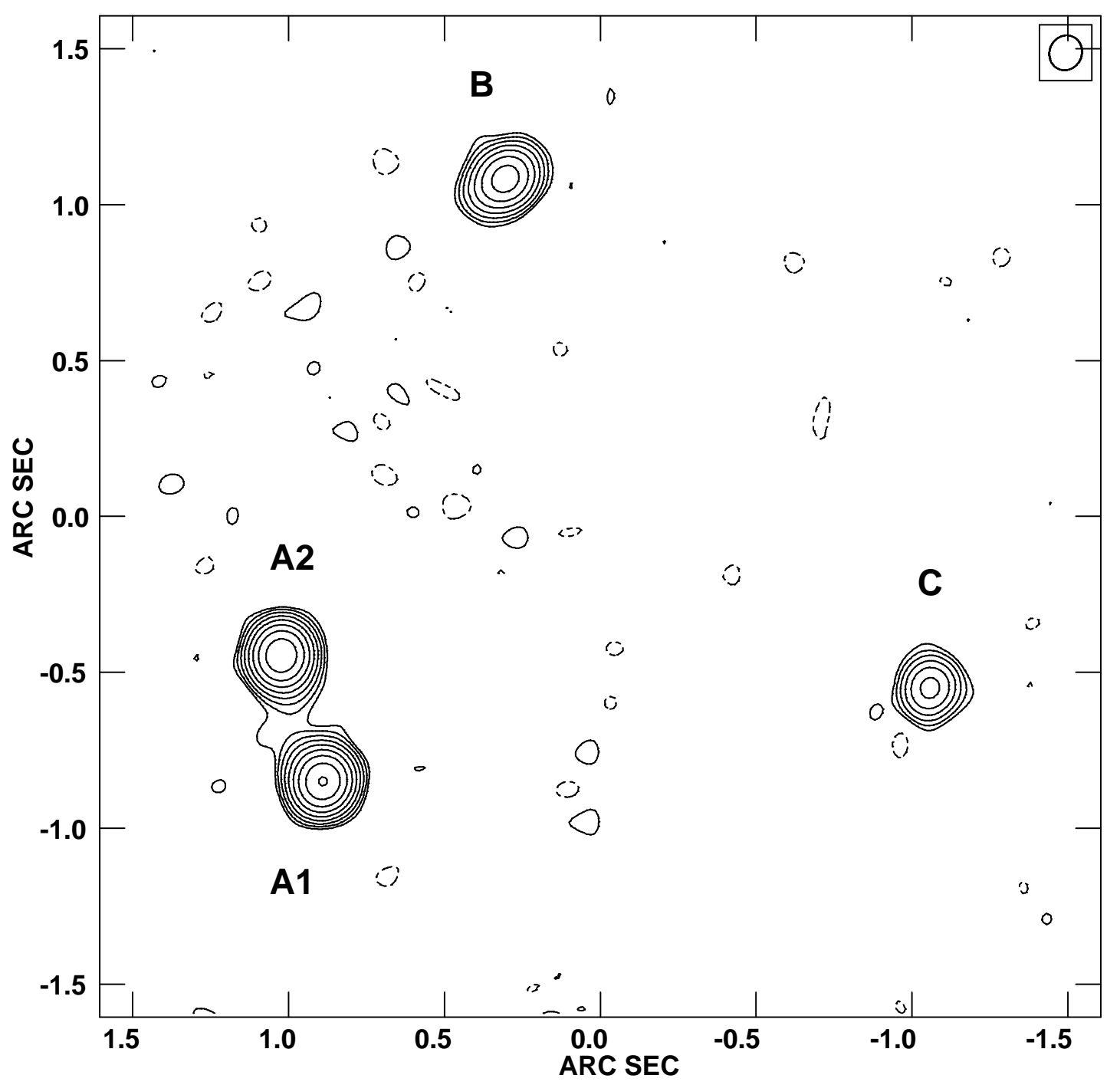

Fig. 4.- Contour plot of $15 \mathrm{GHz}$ map of MG $0414+0534$ on 93 Jan 15. Contour levels are $-0.375 \%$, $0.375 \%, 0.75 \%, 1.5 \%, 3 \%, 6 \%, 12 \%, 24 \%, 48 \%$, and $96 \%$ of the peak intensity of $159 \mathrm{mJy} / \mathrm{beam}$. The RMS noise level is $250 \mu \mathrm{Jy} /$ beam. The box in the upper right corner shows the beam FWHM ellipse. The map is centered at RA $04^{h} 14^{m} 37.708^{s}$, Dec $+05^{\circ} 34^{\prime} 43.19^{\prime \prime}$ (J2000). 


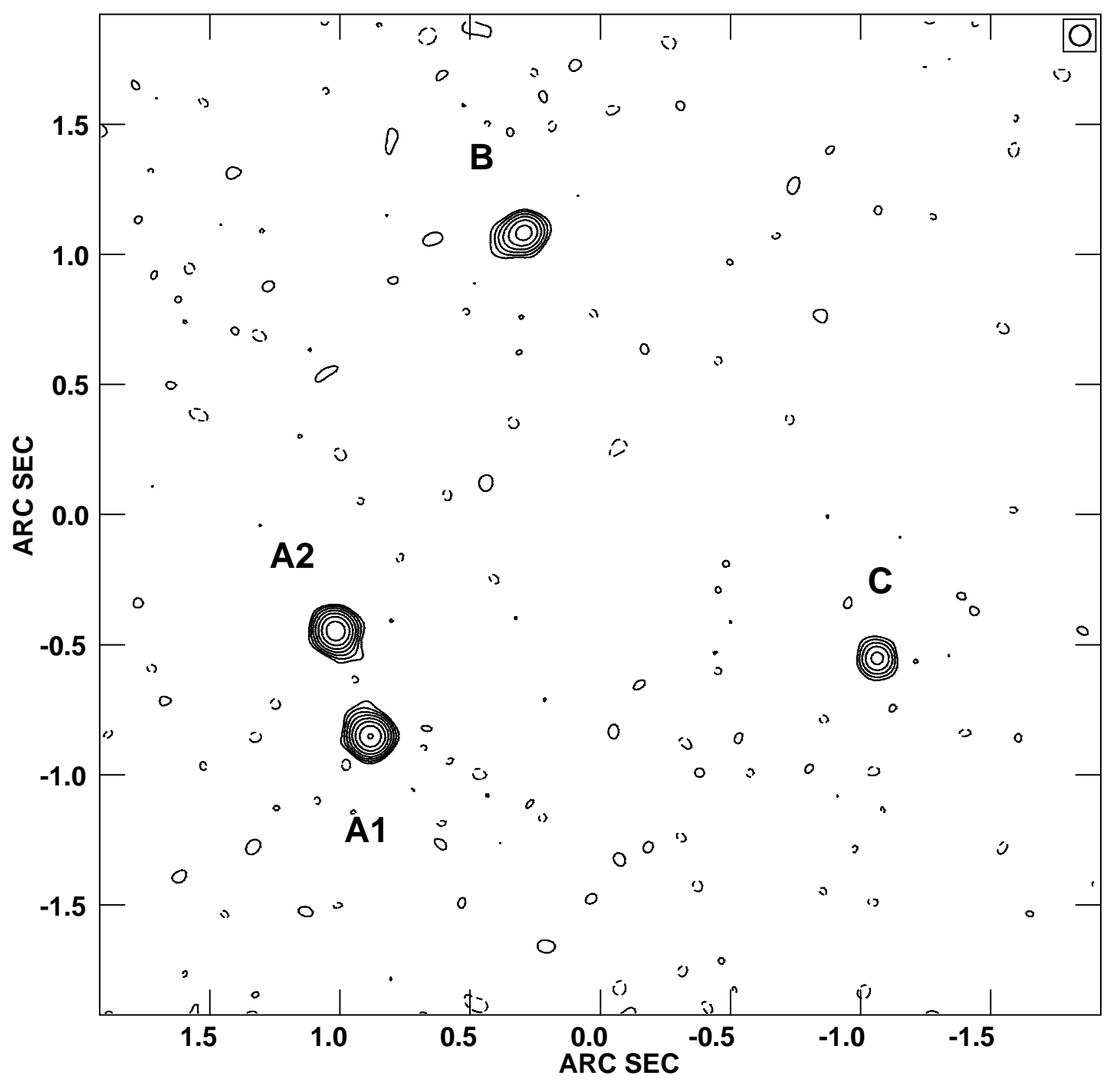

Fig. 5.- Contour plot of $22 \mathrm{GHz}$ map of MG $0414+0534$ on 94 Apr 1. Contour levels are $-0.75 \%$, $0.75 \%, 1.5 \%, 3 \%, 6 \%, 12 \%, 24 \%, 48 \%$, and $96 \%$ of the peak intensity of $112 \mathrm{mJy} /$ beam. The RMS noise level is $330 \mu \mathrm{Jy} /$ beam. The box in the upper right corner shows the beam FWHM ellipse. The map is centered at RA $04^{h} 14^{m} 37.708^{s}$, Dec $+05^{\circ} 34^{\prime} 43.19^{\prime \prime}$ (J2000). 


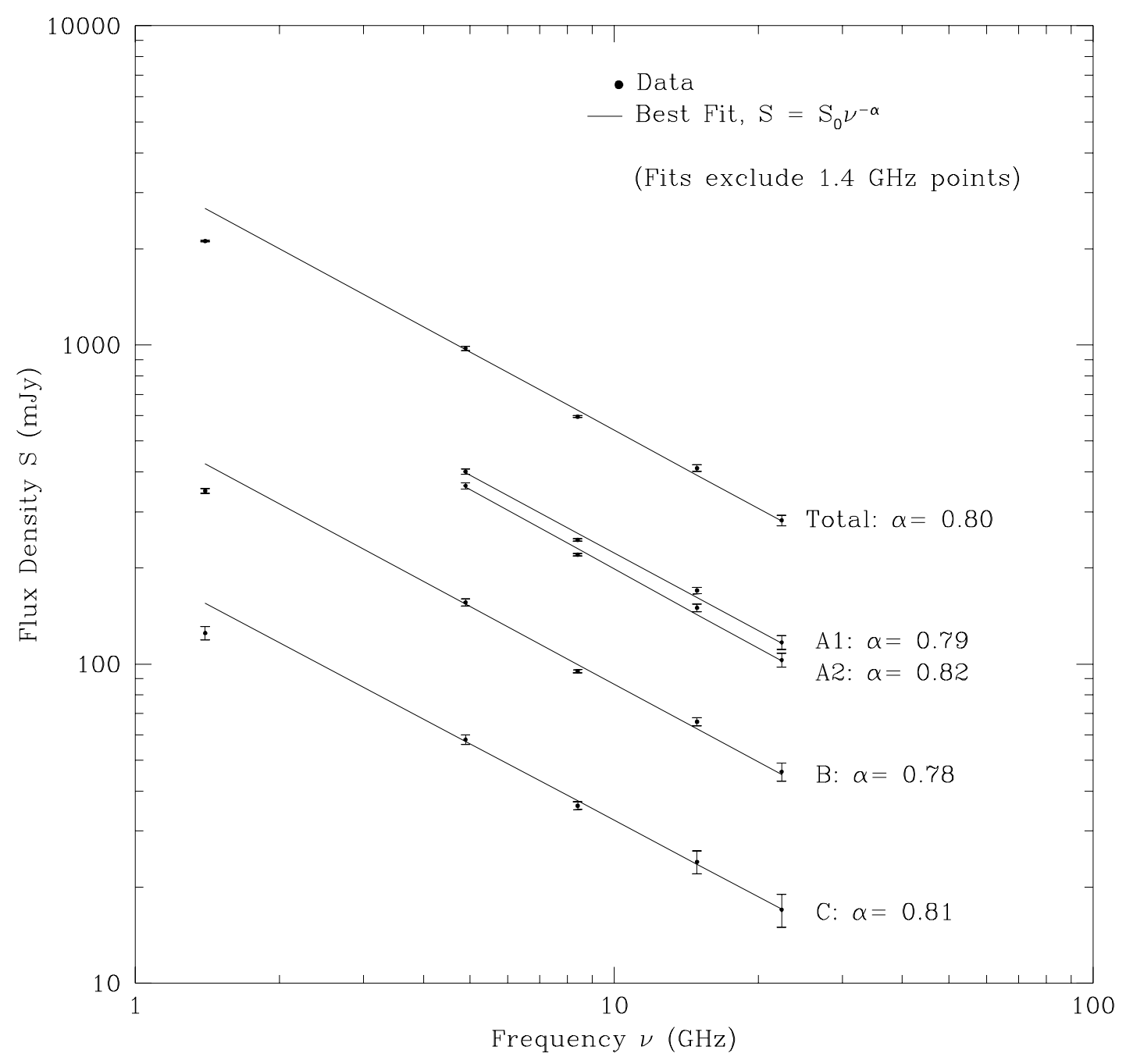

Fig. 6.- Plot of measured flux densities of each component with spectral model fits. The fits exclude the $1.4 \mathrm{GHz}$ data (see text). 


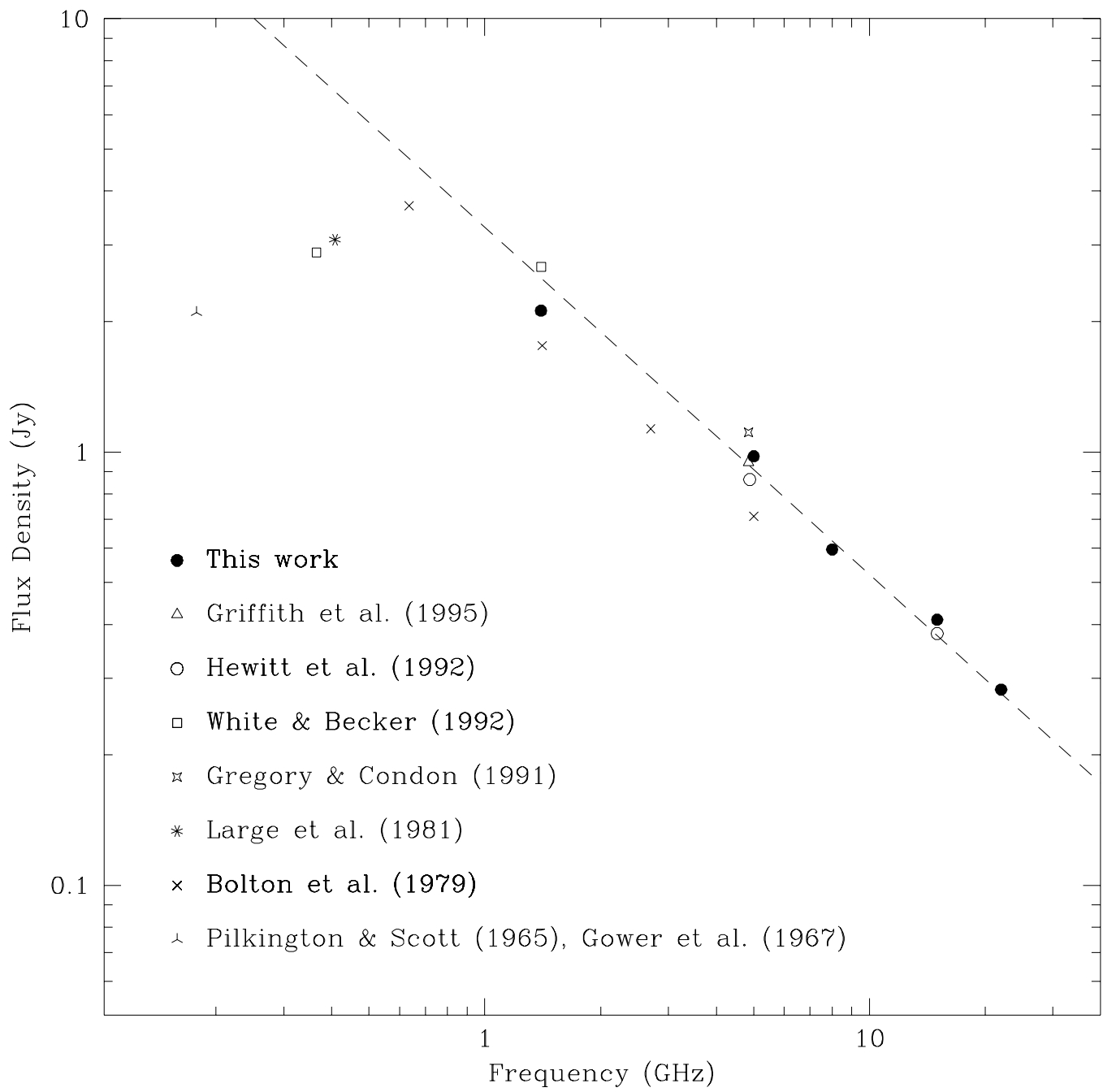

Fig. 7.- MG 0414+0534 total flux density from this work and the literature. The dashed line indicates the power-law fit to data from this work above $1.4 \mathrm{GHz}$ (see text and Figure 6). 